\title{
Different cholinergic cell groups in the basal forebrain regulate social interaction and social recognition memory
}

\section{Kana Okada}

Hiroshima University

Kayo Nishizawa

Fukushima Medical University

Tomoko Kobayashi

Fukushima Medical University

Shogo Sakata

Hiroshima University

Kouichi Hashimoto

Hiroshima University

Kazuto Kobayashi ( $\nabla$ kazuto@fmu.ac.jp )

Fukushima Medical University

\section{Research Article}

Keywords: social interaction, social recognition memory, Alzheimer's disease (AD), dementia

Posted Date: December 10th, 2020

DOI: https://doi.org/10.21203/rs.3.rs-119127/v1

License: () (1) This work is licensed under a Creative Commons Attribution 4.0 International License. Read Full License 


\section{Different cholinergic cell groups in the basal forebrain}

\section{regulate social interaction and social recognition memory}

Kana Okada ${ }^{1,2}$, Kayo Nishizawa ${ }^{3}$, Tomoko Kobayashi ${ }^{3}$, Shogo Sakata ${ }^{4}$, Kouichi

Hashimoto $^{1} \&$ Kazuto Kobayashi ${ }^{3 *}$

${ }^{1}$ Department of Neurophysiology, Graduate School of Biomedical \& Health Sciences, Hiroshima University, Hiroshima 734-8551, Japan

${ }^{2}$ Career Assistant Project, Hiroshima University, Higashi-Hiroshima 739-8521, Japan

${ }^{3}$ Department of Molecular Genetics, Institute of Biomedical Sciences, Fukushima Medical University School of Medicine, Fukushima 960-1295, Japan

${ }^{4}$ Department of Behavioural Sciences, Graduate School of Humanities and Social Sciences, Hiroshima University, Higashi-Hiroshima 739-8521, Japan

*Corresponding Author: Kazuto Kobayashi, Ph.D.

Department of Molecular Genetics, Institute of Biomedical Sciences, Fukushima Medical University School of Medicine, Fukushima 960-1295, Japan

TEL: 81-24-547-1667, FAX: 81-24-548-3936, E-mail: kazuto@fmu.ac.jp 
Social behaviour is a complex construct that is reported to include several components of social approach, interaction and recognition memory. Alzheimer's disease (AD) is mainly characterized by progressive dementia and is accompanied by cognitive impairments, including a decline in social ability. The cholinergic system is a potential constituent for the neural mechanisms underlying social behaviour, and impaired social ability in AD may have a cholinergic basis. However, the involvement of cholinergic function in social behaviour has not yet been fully understood. Here, we performed a selective elimination of cholinergic cell groups in the basal forebrain in mice to examine the role of cholinergic function in social interaction and social recognition memory by using the threechamber test. Elimination of cholinergic neurons in the medial septum (MS) and vertical diagonal band of Broca (vDB) caused impairment in social interaction, whereas ablating cholinergic neurons in the nucleus basalis magnocellularis (NBM) impaired social recognition memory. These impairments were restored by treatment with cholinesterase inhibitors, leading to cholinergic system activation. Our findings indicate distinct roles of MS/vDB and NBM cholinergic neurons in social interaction and social recognition memory, suggesting that cholinergic dysfunction may explain social ability deficits associated with AD symptoms. 
Social behaviour is a complex construct and may include several components for social approach, interaction, and recognition memory [1-3]. Previous studies have reported that social behaviour requires the function of multiple brain regions, such as the medial prefrontal cortex [4-7]. The cholinergic system is a potential constituent for the neural mechanisms underlying social behaviour [8-10]. Indeed, systemic administration of the muscarinic acetylcholine receptor antagonist scopolamine disrupts social interaction or social recognition in rodents [11-13], and deficits in these behaviours were restored by treatment with the acetylcholinesterase inhibitor galantamine or the nicotinic acetylcholine receptor agonist AR-R $17779[12,13]$.

The most prominent characteristic of Alzheimer's disease (AD) is mnemonic dysfunction, but along with the progress of the disease, behavioural and psychological symptoms of dementia (BPSD), including social illness, are commonly seen in patients $[14,15]$. Mnemonic dysfunction in AD is considered to be mostly caused by the substantial loss of acetylcholine-containing neurons in the basal forebrain [16-18], and a number of experimental studies in animals support the finding that basal forebrain cholinergic neurons play a crucial role in mnemonic function $[19,20]$. In addition, previous studies have suggested that some BPSD, including impaired social ability, also appear to have a cholinergic basis $[21,22]$. However, the involvement of basal forebrain cholinergic system in social behaviour is not yet fully understood.

The basal forebrain cholinergic system is comprised of discrete cell groups that innervate a variety of brain regions; neurons in the medial septum (MS) and vertical diagonal band of Broca (vDB) provide cholinergic projections predominantly to the hippocampus and the medial prefrontal cortex, and neurons in the nucleus basalis magnocellularis (NBM) provide cholinergic innervations to the entire cortex and 
basolateral amygdala $[23,24]$. These anatomical findings that cholinergic projection areas overlap with the brain regions required for social behaviour indicate the possibility that basal forebrain cholinergic cell groups may be involved in the control of social function through modulation of the activity of the related brain regions.

To address the role of MS/vDB and NBM cholinergic neurons in the basal forebrain in social behaviour, we carried out immunotoxin (IT)-mediated cell targeting $[25,26]$ to selectively eliminate each cholinergic cell group, and then tested social behaviour by using the three-chamber test that was developed as the standardized assay for the assessment of social interaction and social recognition memory in mice [3]. IT injection into the MS/vDB or NBM selectively removed the respective cholinergic groups in transgenic (Tg) mice. Elimination of MS/vDB cholinergic neurons damaged the preference for social stimuli over non-social stimuli, indicating reduction in social interaction, whereas it did not alter the detection of novel social stimuli, showing the intact social recognition memory. In contrast, NBM cholinergic elimination, although persisted normally social interaction, resulted in impaired social recognition memory. The decline in social interaction and social recognition memory in the injected mice was restored by cholinergic activation with cholinesterase inhibitors (ChEIs), such as donepezil (Done) and rivastigmine (Riva). Our results indicate that MS/vDB and NBM cholinergic neurons have important roles in different types of social behaviour, suggesting that deficits in basal forebrain cholinergic systems may explain impairment in social ability related to AD symptoms. 


\section{Results}

Selective targeting of MS/vDB and NBM cholinergic cell groups. We performed

selective elimination of cholinergic neurons in the basal forebrain by using IT-mediated cell targeting $[25,26]$. Tg mice were generated that carry a chimeric gene encoding human intelekin-2 receptor $\alpha$-subunit (IL-2R $\alpha$ ) fused to a variant of enhanced yellow fluorescent protein (mVenus) under the control of the gene promoter for choline acetyltransferase (ChAT), and the IL-2R $\alpha /$ mVenus transgene was expressed in the majority of ChAT-positive neurons in both the MS/vDB and NBM regions of the ChAT$I L-2 R \alpha / m V e n u s$ mice, as described in our previous study [20]. Anti-Tac(Fv)-PE38, which consists of single-chain variable regions of a monoclonal antibody for human IL$2 \mathrm{R} \alpha$ connected to a bacterial exotoxin catalytic fragment [27], was used as a recombinant IT for intracranial injection into the basal forebrain regions (Fig. 1a, b). Tg and non-transgenic (non-Tg) mouse littermates (8-10 weeks old) were given intracranial injections of IT $(20 \mu \mathrm{g} / \mathrm{ml})$ or phosphate-buffered saline (PBS) into the $\mathrm{MS} / \mathrm{vDB}(0.2 \mu \mathrm{l} \times 12$ sites $)$ or NBM $(0.3 \mu \mathrm{l} \times 6$ sites $)$. One week after the surgery, the brains were processed for immunohistochemistry. Sections through the basal forebrain were immunostained by using anti-ChAT antibody, and were viewed for cell counts. IT injection into the MS/vDB or NBM resulted in a loss of ChAT-positive neurons in the corresponding regions in the $\mathrm{Tg}$ mice only (Fig. 1c, d). One-way analysis of variance (ANOVA) for the mice that received injection into the MS/vDB indicated a significant difference in the number of cells in the MS/vDB among the four mouse groups (Fig. 1e; $\left.F_{3,8}=26.567, P<0.001\right)$, with a significant reduction in the IT-injected Tg mice compared with each of other three groups (Bonferroni method, $P<0.05$ ). However, no significant difference in the cell number in the NBM was observed among the mouse 
groups (Fig. 1e). For the mice injected into the NBM, one-way ANOVA followed by post hoc multiple comparisons revealed that the NBM cell number was significantly lower in the IT-injected Tg mice compared with those in each of other three groups (Fig. 1d; $F_{3,8}=11.316, P=0.003$, Bonferroni method, $\left.P<0.05\right)$, whereas there was no difference in the MS/vDB cell number among the groups (Fig. 1f). These findings confirm the selective and efficient elimination of cholinergic neurons in the basal forebrain regions of the Tg mice after IT injection.

\section{Targeting MS/vDB and NBM neurons impairs different types of social behaviour.}

Tg and non-Tg mice (8-10 weeks old) were administered PBS or IT injection into the $\mathrm{MS} / \mathrm{vDB}$ or NBM, and were subjected to the three-chamber test that was developed as a standardized assay for the assessment of social interaction and social recognition memory [3]. The test was carried out using apparatus that consisted of three open-field adjacent chambers (a central chamber and two side chambers) separated by two clear plastic dividers, and connected by open doorways (Fig. 2a). The procedure consisted of three 10-min sessions for (i) familiarization, (ii) social interaction, and (iii) social recognition memory with 1-min intersession intervals. In the first session, familiarization (Fig. 2a-i), the mice were placed in the central chamber, allowed to habituate to the apparatus, and freely explored it. To monitor the locomotor activity of the mouse groups, the travelled distance in the apparatus was measured by using a video tracking system. The distance was similar among the four mouse groups that received the MS/vDB injection (Fig. $2 \mathrm{~b}: F_{3,56}=0.956, P=0.420$, one-way ANOVA) or NBM injection (Fig. 2c: $F_{3,56}=1.461, P=0.235$, one-way ANOVA), showing normal 
locomotor activity during the familiarization session in the mice with elimination of the $\mathrm{MS} / \mathrm{vDB}$ or NBM cell group.

In the second session for social interaction (Fig. 2a-ii), a steel meshed cage with an unfamiliar C57BL/6J male mouse (8-10 weeks old) was placed in one of two side chambers (mouse chamber), and another identical empty cage was placed as an object in the other side chamber (object chamber). The duration of how long an injected mouse would stay in each chamber was measured, and the value in the mouse chamber against the object chamber was compared, because animals normally prefer to explore social stimuli than objects. To alleviate the influence of baseline locomotor activity variability among individual animals on the analysis of social behaviours, the data were normalized as z-scores according to the formula: $z=\frac{X-\bar{X}}{S}$, where $X$ was the raw score of duration in each chamber, $\bar{X}$ is the mean among the durations in all chambers, and $S$ is the standard deviation. For the MS/vDB injection, the duration in the chamber in the three mouse groups except for the IT-injected Tg mice showed higher scores for the mouse chamber compared to those for the object chamber. The scores were not significantly different between the two chambers in the IT-injected Tg group only (Fig. 2d: group, $F_{3,56}=0.519, P=0.671$; chamber, $F_{1,56}=23.692, P<0.001$; interaction, $F_{3,56}$ $=3.566, P=0.020$, two-way ANOVA; non-Tg/PBS, $F_{1,56}=20.331, P<0.001$; non$\mathrm{Tg} / \mathrm{IT}, F_{1,56}=7.679, P=0.008 ; \mathrm{Tg} / \mathrm{PBS}, F_{1,56}=9.380, P=0.003 ; \mathrm{Tg} / \mathrm{IT}, F_{1,56}<0.001, P$ $=0.992$, post hoc). For the NBM injection, the duration in the four mouse groups showed the larger scores for the mouse chamber compared to the object chamber (Fig. 2e: group, $F_{3,56}=2.037, P=0.119$; chamber, $F_{1,56}=44.702, P<0.001$; interaction, $F_{3,56}$ $=1.017, P=0.392$, two-way ANOVA). These data suggest that elimination of the 
MS/vDB cholinergic cell group resulted in impairment of social interaction, whereas NBM cholinergic ablation did not influence the interaction in the three-chamber test. In the third session, for social recognition memory (Fig. 2a-iii), the cage with the male mouse in the second session was kept in the same side chamber (old mouse chamber), in which the mouse becomes familiar one. Another identical cage with a new, unfamiliar C57BL/6J male mouse (8-10 weeks old) was placed in the side chamber (new mouse chamber) as opposed to the empty cage that was used in the second session. The duration in the chamber of the injected mouse was monitored, and the preference for the new mouse chamber over the old mouse chamber was compared, since animals normally prefer to explore novel social stimuli than familiar social stimuli, depending on mnemonic function to differentiate between these two stimuli. As in the second session, the duration data were normalized as z-scores. For the MS/vDB injection, chamber durations in the four mouse groups exhibited higher scores for the new mouse chamber compared to the old mouse chamber (Fig. 2f: group, $F_{3,56}=1.068$, $P=0.370$; chamber, $F_{1,56}=39.066, P<0.001$; interaction, $F_{3,56}=0.528, P=0.665$, twoway ANOVA). For the NBM injection, the duration in the mouse groups other than the IT-injected Tg mouse group had greater scores for the new mouse chamber compared to the old mouse chamber, but there was no significant difference in the scores between the two chambers in the IT-injected Tg group (Fig. $2 \mathrm{~g}$ : group, $F_{3,56}=1.676, P=0.183$; chamber, $F_{1,56}=43.599, P<0.001$; interaction, $F_{3,56}=5.902, P=0.001$, two-way ANOVA; non-Tg/PBS, $F_{1,56}=14.993, P<0.001$; non-Tg/IT, $F_{1,56}=28.925, P<0.001$; $\mathrm{Tg} / \mathrm{PBS}, F_{1,56}=17.355, P<0.001 ; \mathrm{Tg} / \mathrm{IT}, F_{1,56}=0.044, P=0.835$, post hoc $)$. These results suggest that cholinergic elimination of the NBM cell group impairs social 
recognition memory, while sustaining recognition memory for MS/vDB cholinergic removal.

Our findings obtained from the behavioural analysis with the three-chamber test demonstrate the functional double dissociation of basal forebrain cholinergic cell groups in social behaviour, revealing distinct roles of cholinergic neurons in the MS/vDB and NBM in social interaction and social recognition memory, respectively.

\section{Impaired social behaviour is recovered by pharmacological cholinergic activation.}

We examined whether impairment in social interaction or social recognition memory of mice lacking basal forebrain cholinergic cell groups could be recovered by pharmacological cholinergic activation with ChEIs that inhibit the degradation of acetylcholine, including Done and Riva. These drugs are known to be anti-dementia agents for $\mathrm{AD}$ [28-31], and proved to be effective on the memory deficits in AD models of rodents $[20,32]$. After the bilateral IT injection into the MS/vDB or NBM, Tg and non-Tg mice were administered intraperitoneally (i.p.) saline or low and high doses of Done and Riva ( 1 and $4 \mu \mathrm{mol} / \mathrm{kg}$, respectively), and 30 min later they were subjected to the three-chamber test.

The mice that received IT-injection into the MS/vDB were tested for the recovery of social interaction after the familiarization session (Fig. 3a). Following saline administration, the duration in the mouse chamber was significantly longer than that in the object chamber in the non-Tg mice, whereas the scores were similar between the two chambers in the Tg mice (Fig. $3 b$ : group, $F_{1,18}=1.244, P=0.279$; chamber, $F_{1,18}=$ $0.149, P=0.704$; interaction, $F_{1,18}=7.832, P=0.502$, two-way ANOVA; non-Tg, $F_{1,18}$ $=5.072, P=0.037 ; \mathrm{Tg}, F_{1,18}=2.909, P=0.105$, post hoc), confirming impaired social 
interaction in the Tg mice lacking the MS/vDB cholinergic neurons. After Done administration with either a low or high dose, the duration in the mouse chamber was significantly longer than that in the object chamber in both the non-Tg and Tg mice (Fig 3b: group, $F_{1,18}=0.563, P=0.463$; chamber, $F_{1,18}=8.998, P=0.008$; interaction, $F_{1,18}$ $=2.220, P=0.154$, two-way ANOVA for the low-dose; group, $F_{1,18}=2.980, P=0.101$; chamber, $F_{1,18}=20.46, P<0.001$; interaction, $F_{1,18}=2.624, P=0.123$, two-way ANOVA for the high-dose group). After the low-dose Riva administration, the duration was significantly longer for the mouse chamber compared to the object chamber in the non-Tg mice, but there was no significant difference between the two chambers in the Tg mice (Fig. 3b: group, $F_{1,18}=0.697, P=0.339$; chamber, $F_{1,18}=1.557, P=0.229$; interaction, $F_{1,18}=5.539, P=0.030$, two-way ANOVA; non-Tg; $F_{1,18}=6.476, P=$ $0.020 ; \mathrm{Tg}, F_{1,18}=0.614, P=0.443$, post hoc $)$. Following the high-dose Riva administration, the duration was significantly longer in the mouse chamber compared to that in the object chamber in both kinds of mice (Fig. 3b: group, $F_{1,18}=0.113, P=0.741$; chamber, $F_{1,18}=4.512, P=0.048$; interaction, $F_{1,18}=0.064, P=0.803$, two-way ANOVA). These results indicate that the administration of ChEIs, except in the mice administered with a low dose of Riva, can restore the decline in social interaction generated by the dysfunction of MS/vDB cholinergic cell group.

The mice that received IT injection into the NBM were tested for the reversal of social recognition memory after the familiarization and social interaction sessions (Fig. 4a). Following saline administration, the duration in the new mouse chamber was significantly longer than that in the old mouse chamber in the non-Tg mice, while the values were similar between the two chambers in the Tg mice (Fig. $4 \mathrm{~b}$ : group, $F_{1,18}=$ $0.287, P=0.599 ;$ chamber, $F_{1,18}=2.77 .1, P=0.161 ;$ interaction, $F_{1,18}=4.861, P=$ 
0.041, two-way ANOVA; non-Tg, $F_{1,18}=6.723, P=0.018$; $\mathrm{Tg}, F_{1,18}=0.276, P=0.606$, post hoc), ascertaining the impaired social recognition memory in the $\mathrm{Tg}$ mice deleting NBM cholinergic neurons. After the low-dose Done administration, the duration in the new mouse chamber was significantly longer than that in the old mouse chamber in the non-Tg mice, but there was no significant difference in duration between the two chambers in the Tg mice (Fig. 4b: group, $F_{1,18}=1.790, P=0.198$; chamber, $F_{1,18}=$ 5.812, $P=0.027$; interaction, $F_{1,18}=4.446, P=0.049$, two-way ANOVA; non-Tg, $F_{1,18}$ $=10.212, P=0.005 ; \mathrm{Tg}, F_{1,18}=0.046, P=0.833$, post hoc $)$. After the high-dose Done administration, the duration was significantly longer for the new mouse chamber compared to the old mouse chamber in both the non-Tg and Tg mice (Fig. 4b: group, $F_{1,18}=0.128, P=0.725$; chamber, $F_{1,18}=11.865, P=0.003$; interaction, $F_{1,18}=0.174, P$ = 0.682; two-way ANOVA). Following Riva administration with the low/high doses, the duration in the new mouse chamber was significantly longer than that in the old chamber in both kinds of mice (Fig. 4 b: group, $F_{1,18}=21.775, p<0.001$; chamber, $F_{1,18}$ $=14.737, P=0.001$; interaction, $F_{1,18}=0.167, P=0.201$, two-way ANOVA for low dose; group, $F_{1,18}=0.752, P=0.397$; chamber, $F_{1,18}=8.845, P=0.008$; interaction, $F_{1,18}=0.948, P=0.343$, two-way ANOVA for high dose). These data indicate that, although the low dose of Done was ineffective, the administration of ChEIs can reverse disturbance in social recognition memory produced by NBM cholinergic dysfunction.

\section{Impact of cholinesterase inhibition on locomotor activity in mice lacking}

cholinergic cell groups. Additionally, we examined the effect of systemic ChEI administration (i.p.) on the locomotor activity of the $\mathrm{Tg}$ and non- $\mathrm{Tg}$ mice with IT injection into the MS/vDB or NBM. To evaluate the locomotor activity of the mouse 
groups, we measured the travelled distance during the familiarization session of the mice used for the recovery experiments mentioned above. For the mice that were IT injection into the MS/vDB, the travelled distance was comparable among the mouse groups that were administered saline, low/high doses of Done, and low dose of Riva. The distance was significantly reduced only in the mice administered a high dose of Riva compared to saline administration, although it did not differ between the Tg and non-Tg mice with all conditions of the administration (Fig. 5a: group, $F_{1,90}=0.008, P=$ 0.928 ; drug, $F_{4,90}=10.924, P<0.001$; interaction, $F_{4,90}=1.747, P=0.147$, two-way ANOVA, Bonferroni method, $P<0.05)$. For the mice that received IT injection into the NBM, similarly to the MS/vDB injection, the travelled distance was indistinguishable among the administration with saline, low/high doses of Done, and low dose of Riva. In addition, the distance was significantly decreased only in the administration with the high dose of Riva compared to saline administration, but there was no difference in the distance between the two kinds of mice with all administration conditions (Fig. 5b: group, $F_{1,90}=2.879, P=0.093$; drug, $F_{4,90}=36.167, P<0.001$; interaction, $F_{4,90}=1.799, P=0.136$, two-way ANOVA, Bonferroni method, $\left.P<0.05\right)$. These data show that locomotor activity in the apparatus during the familiarization session was decreased in the mice only when the high dose of Riva was administered, regardless of the presence or absence of cholinergic neurons in the MS/vDB or NBM, suggesting that high-dose administration of Riva may have a side effect of reducing locomotion in mice. 


\section{Discussion}

In the present study, we aimed to clarify the roles of basal forebrain cholinergic cell groups in social behaviour. We performed selective targeting of MS/vDB and NBM cholinergic cell groups in mice with IT-mediated cell targeting, and examined the social behaviour of these mice by using the three-chamber test. Selective elimination of $\mathrm{MS} / \mathrm{vDB}$ cholinergic neurons resulted in no preference for the chamber with the social stimulus (mouse) over that with the non-social stimulus (object), indicating impairment in social interaction. The elimination of NBM cholinergic neurons displayed no preference to the chamber with the novel social stimulus (new mouse) against the familiar one (old mouse), implying the impaired social recognition memory. The deficit in social interaction or social recognition memory by dysfunction of different cholinergic cell groups was restored by systemic administration with appropriate doses of ChEIs including Done and Riva that activate the cholinergic systems. Our results reveal that MS/vDB and NBM cholinergic cell groups have critical and dissociable roles in social interaction and social recognition memory, respectively.

Our cell targeting technology with anti-Tac(Fv)-based recombinant IT enabled us to conduct the selective, efficient elimination of basal forebrain cholinergic neurons without any influence on the number of parvalbumin-positive GABAergic neurons and tissue damage around the injection and the elimination of these neurons did not affect the locomotor activity [20]. In contrast, other previous studies reported that 192 IgGsaporin and p75-conjugated saporin occasionally induce non-specific damage of GABAergic neurons and extensive tissue injury in the basal forebrain [33-36], in addition to producing the limited lesion of neurons [37-39]. Some of these studies also reported that cholinergic ablation with saporin-conjugated neurotoxins leads to an 
increase in locomotion [39-42]. The inconsistency in locomotor activity after cholinergic ablation between previous studies and the current study suggests that nonspecific damages in the basal forebrain regions affected locomotor activity in the previous studies.

In this study, although systemic administration of high doses of Riva recovered impaired social interaction or social recognition memory in mice lacking different cholinergic cell groups in the basal forebrain, this treatment generated a decrement in locomotor activity in both the $\mathrm{Tg}$ and non- $\mathrm{Tg}$ mice that received IT injection, suggesting the potential of high-dose Riva to reduce locomotion, irrespective of the presence or absence of cholinergic neurons. One possible explanation for this observation is that the systemic administration may influence the activity of cholinergic interneurons in the striatum, and that these interneurons may be more susceptible to high doses of Riva. Ablation of striatal cholinergic interneurons is known to cause hyperactivity in mice, suggesting an inhibitory role of these neurons in locomotion [43, 44]. Therefore, striatal cholinergic activation may be involved in the hypoactivity observed after systemic administration of high-dose Riva. Another possibility may be related to the trait on behavioural effects of butyrylcholinesterase, which is inhibited selectively by Riva. Both Done and Riva inhibit acetylcholinesterase, and Riva possesses an additional inhibitory activity against butyrylcholinesterase [45]. Butyrylcholinesterase-positive neurons are found in the forebrain and brain stem, including the thalamus and tegmental areas $[46,47]$. The thalamus and tegmental areas constitute parts of the cortico-basal ganglia loop, which plays a central role in the control of locomotor activity $[48,49]$. The effect of high-dose Riva may influence, partially through the inhibition of butyrylcholinesterase, the activity of the cortico-basal 
ganglia loop, leading to reduced locomotor activity; however, the neural mechanism that explains butyrylcholinesterase inhibition affecting the cortico-basal ganglia circuit remains unknown.

Our findings from the three-chamber test show that MS/vDB cholinergic neurons are involved in social interaction but are not involved in social recognition memory. There are prominent anatomical connections between MS/vDB cholinergic neurons and the dorsal and ventral hippocampus $[23,50]$. Social interaction is reported to be increased by excitotoxic lesion of the whole hippocampal region [51] and, especially, the ventral hippocampus in rats [52]. Lesion of the fimbria results in a reduction of social interdependency without affecting affiliative behaviours such as grooming, sniffing, and nosing [5]. These findings suggest that cholinergic activation in the septohippocampal pathway may mediate the processing of social information during social interaction. In addition, social interaction is reported to be regulated by cholinergic activity in the prefrontal cortex [53]. It is possible that cortical cholinergic activities may affect the hippocampal function in different types of social contacts interacting with the septo-hippocampal pathway. More detailed examination of the roles of cholinergic cell groups in various types of social interactions will provide clues for the elucidation of the neural mechanisms underlying social ability in mammals. In contrast, ablation of cholinergic NBM neurons in rats by 192 IgG-saporin decreases the passive social contact, such as sitting or lying closely to each other, but increases active social contact as sniffing, grooming, and fighting [54]. This result shows the involvement of NBM cholinergic neurons in social contacts, which is not consistent with our results, which show the independency of NBM cholinergic lesions in social interaction. One reason that explains the inconsistency is because of the difference in the monitoring 
methods of social behaviour. Social interaction was evaluated by comparing relative duration in a chamber with a mouse with a chamber with an object in the current study, whereas it was monitored by examining multiple factors constituting social contacts in their study [54]. Alternatively, non-specific neuronal damages in the NBM after local injection of 192 IgG-saporin may have led to the complex changes in social contacts.

In contrast to the MS/vDB, NBM cholinergic neurons were engaged in social recognition memory, but not in social interaction in the present study. Our findings support those of previous studies, which reported the damage of social recognition memory by systemic dysfunction of cholinergic system $[55,56]$. The cholinergic terminals of NBM widely distribute to various neocortical regions as well as the amygdala [23, 57], which are involved in social recognition memory [58]. These studies support that cholinergic NBM projections may mediate the information processing during social recognition. In addition to social recognition memory, NBM cholinergic neurons are also required for object recognition memory [20]. These requirements suggest that dysfunction of the NBM cholinergic system generally impairs recognition memory depending on various cues. It is unknown whether different types of cholinergic neurons in the NBM are involved in social and object recognition memory. Further investigation will be needed to identify how this cholinergic cell group regulates processing for specific types of recognition memory.

Early mnemonic impairments in AD are considered to mainly be caused by the dysfunction of cholinergic neurons [16-18], but it is still uncertain whether the loss of cholinergic neurons in the basal forebrain is related to BPSD. Nonetheless, BPSD is an important clinical target for $\mathrm{AD}$ intervention [59]. In the present study, the elimination of cholinergic cell groups on MS/vDB and NBM in the basal forebrain differently 
resulted in the impairments of social behaviour which were restored by treatment with ChEIs. These data suggest that dysfunction of the cholinergic system in the basal forebrain may explain deficits in social ability associated with BPSD in AD. Our strategy provides a new Tg animal model for BPSD based on the selective loss of basal forebrain cholinergic neurons, not only for the dementia with recognition memory impairment [20]. This animal model will be useful for the elucidation of the neural mechanisms underlying cognitive impairments and for the development of approaches for diagnostic and therapeutic treatments.

\section{Methods}

Animals. ChAT-IL-2R $\alpha / m$ Venus $\mathrm{Tg}$ mice that carried a gene cassette encoding IL-2R $\alpha / m V e n u s$ followed by the SV40 early-gene polyadenylation signal downstream of the murine ChAT gene promoter were generated, and the $\mathrm{Tg}$ mie were identified using Southern blot hybridization or PCR with genomic DNA prepared from tail clips as described in our previous study [20]. The Tg and non-Tg littermates were used for the following experiments. All animal experiments were approved and performed in accordance with the Guidelines for the Care and Use of Laboratory Animals established by the Animal Experiments Committee of Fukushima Medical University and Hiroshima University.

Intracranial injection. Surgery was conducted as described by Okada et al. [20]. Mice ( $8-10$ weeks old) were anesthetized with sodium pentobarbital (50 mg/kg, i.p.) and subjected to bilateral intracranial injection of IT solution $[20 \mu \mathrm{g} / \mathrm{ml}$ anti-Tac(Fv)PE38 in PBS containing 0.1\% mouse serum albumin]. For targeting of cholinergic neurons in the MS/vDB and NBM, IT solution or vehicle was injected into 12 sites $(0.2$ 
$\mu 1 /$ site $)$ and six sites $(0.3 \mu 1 /$ site $)$, respectively, through a glass micropipette that was stereotaxically introduced by using the coordinates from an atlas of the mouse brain [60]. The anteroposterior, mediolateral and dorsoventral coordinates $(\mathrm{mm})$ from bregma and dura were $(1.1, \pm 0.1,-3.7),(1.1, \pm 0.1,-4.1),(0.8, \pm 0.1,-3.8),(0.8, \pm 0.3,-4.7)$, $(0.6, \pm 0.1,-3.7)$, and $(0.6, \pm 0.1,-4.2)$ for injection into the MS/vDB; and $(-0.4, \pm 1.6$, $-3.7),(-0.7, \pm 1.8,-3.8)$, and $(-0.9, \pm 2.0,-3.8)$ for injection into the NBM. Injection was carried out at a constant flow rate of $0.1 \mu \mathrm{l} / \mathrm{min}$ with a microinfusion pump (ESP36, EICOM), and the micropipette was left in situ for 2 min after each infusion.

Drug treatment. Drug solution was prepared as described previously [20]. Donepezil hydrochloride (Sequoia Research Products Ltd.) and rivastigmine hydrogen tartrate (kindly provided by Novartis Pharma AG, Basel, Switzerland) were dissolved into saline at final concentrations of 0.1 and $0.4 \mathrm{mM}$. The mice received the i.p. treatment of each drug solution ( 1 or $4 \mu \mathrm{mol} / \mathrm{kg}$ ) $30 \mathrm{~min}$ before the behavioural testing.

Histology. Immunohistochemistry and cell counts were performed as described [20]. Fixed brains were cut into sections $(30-\mu \mathrm{m}$ thick), and the sections were incubated with primary antibodies for ChAT (mouse, 1:1,000, Millipore), and then with a biotinylated secondary antibody (anti-mouse IgG, 1:200, Jackson ImmunoResearch Laboratories). The immunoreactive signals were visualized by using a Vectastain Elite $\mathrm{ABC}$ kit (Vector Laboratories). For cell counts, the number of immune-positive cells in each area was counted in the representative four sections through the MS/VDB or NBM (the anteroposterior coordinates from bregma: $1.3,0.9,0.7$, and $0.5 \mathrm{~mm}$ for the MS/VDB; and $-0.3,-0.5,-0.8$, and $-1.0 \mathrm{~mm}$ for the NBM), and the total number of immunopositive cells was calculated. 
Behavioural analysis. The three-chamber test were conducted as described by Moy et al. [3] with some modifications. Adult naïve male mice were housed in standard lab Plexiglas cages $(225 \times 338 \times 140 \mathrm{~mm}$, length $\times$ width $\times$ height, four mice per cage $)$ on a 12-h light/12-h dark cycle. The experiments were conducted during the light period. After the surgery, the mice were given a 1-week recovery period. The experiment was conducted in a room with an illuminance level of 20 lux. The testing apparatus was made of clear Plexiglas and consisted of three open adjacent chambers, including a central chamber and two side chambers (each $200 \mathrm{~mm}$ length $\times 400 \mathrm{~mm}$ width $\times 225 \mathrm{~mm}$ height) separated by two clear plastic dividers and connected by open doorways ( $50 \mathrm{~mm}$ width $\times 85 \mathrm{~mm}$ height). Each animal's behaviour was monitored using an overhead colour CCD camera (ALDP-292P, NADATEL Co., Ltd) mounted on the ceiling, approximately $740 \mathrm{~mm}$ above the field surface. The apparatus and cages were cleaned with $70 \%$ ethanol between animals.

The three-chamber test consisted of three 10-min sessions with 1-min intersession intervals. In the first session, for familiarization (Fig. 2a-i), the mice were allowed to habituate to the apparatus and freely explore it. Additionally, general locomotor activity was measured by the travelled distance in all chambers during this session. In the second session, for social interaction (Fig. 2a-ii), an unfamiliar C57BL/6 J male mouse (8-10 weeks old) was placed in a cylindrical steel-meshed cage $(87 \mathrm{~mm}$ in height $\times 80 \mathrm{~mm}$ in diameter) in one of the side chambers (mouse chamber) and another identical empty cage (object chamber) was placed in the chamber on the other side. The positions of the mouse and object (empty cage) were counterbalanced between the subjects. The experimental mouse subjects freely explored freely all three chambers, and the measurements were the time durations they spent in each chamber. Preference 
for the mouse chamber over the object chamber was set to the index of social interaction. To alleviate the effect of the variation of baseline locomotor activity among individual animals, the data of the durations in each chamber were normalized into zscores, according to the formula: $z=\frac{X-\bar{X}}{S}$, where $X$ is the raw score of duration in each chamber, $\bar{X}$ is the mean among the durations in all chambers, and $S$ is the standard deviation. In the third session, for social recognition memory (Fig. 2a-iii), the cage with the male mouse in the second session was kept in the same side chamber (old mouse chamber), and the empty cage was replaced by an identical cage with another unfamiliar C57BL/6J male mouse (8-10 weeks old) (new mouse chamber). The experimental mouse subjects were again allowed to explore all chambers freely, and the duration in each chamber was measured. Preference for the new mouse chamber over the old mouse chamber was set to the index of social recognition memory. Individual data were also converted to z-scores, as described above. The travelled distance in the apparatus and duration in each chamber were quantified by computer software (Smart 3; Panlab, S.L.U.).

Statistical analysis. For statistical comparisons, ANOVA, followed by post hoc analysis and Bonferroni test, was used with statistical significance set at $P<0.05$. Twoway ANOVA with repeated measure was used for the analysis of within-subjects design. All data were displayed as mean \pm s.e.m. 


\section{REFERENCES}

1. Gabor, C. S., Phan, A., Clipperton-Allen, A. E., Kavaliers, M. \& Choleris, E. Interplay of oxytocin, vasopressin, and sex hormones in the regulation of social recognition. Behav. Neurosci. 126, 97-109, doi:10.1037/a0026464 (2012).

2. Lai, W. S., Ramiro, L. L., Yu, H. A. \& Johnston, R. E. Recognition of familiar individuals in golden hamsters: a new method and functional neuroanatomy. $J$. Neurosci. 25, 11239-11247, doi:10.1523/jneurosci.2124-05.2005 (2005).

3. Moy, S. S. et al. Sociability and preference for social novelty in five inbred strains: an approach to assess autistic-like behavior in mice. Genes Brain Behav. 3, 287302, doi:10.1111/j.1601-1848.2004.00076.x (2004).

4. Felix-Ortiz, A. C., Burgos-Robles, A., Bhagat, N. D., Leppla, C. A. \& Tye, K. M. Bidirectional modulation of anxiety-related and social behaviors by amygdala projections to the medial prefrontal cortex. Neuroscience 321, 197-209, doi:10.1016/j.neuroscience.2015.07.041 (2016).

5. Maaswinkel, H., Gispen, W. H. \& Spruijt, B. M. Executive function of the hippocampus in social behavior in the rat. Behav. Neurosci. 111, 777-784, doi:10.1037//0735-7044.111.4.777 (1997).

6. Paine, T. A., Swedlow, N. \& Swetschinski, L. Decreasing GABA function within the medial prefrontal cortex or basolateral amygdala decreases sociability. Behav. Brain Res. 317, 542-552, doi:10.1016/j.bbr.2016.10.012 (2017).

7. Rilling, J. et al. A neural basis for social cooperation. Neuron 35, 395-405, doi:10.1016/s0896-6273(02)00755-9 (2002). 
8. Martin, L. M. \& Sayette, M. A. A review of the effects of nicotine on social functioning. Exp. Clin. Psychopharmacol. 26, 425-439, doi:10.1037/pha0000208 (2018).

9. Tsuno, N. Donepezil in the treatment of patients with Alzheimer's disease. Expert Rev. Neurother. 9, 591-598, doi:10.1586/ern.09.23 (2009).

10. Vanderschuren, L. J., Niesink, R. J. \& Van Ree, J. M. The neurobiology of social play behavior in rats. Neurosci. Biobehav. Rev. 21, 309-326 (1997).

11. Riedel, G., Kang, S. H., Choi, D. Y. \& Platt, B. Scopolamine-induced deficits in social memory in mice: reversal by donepezil. Behav. Brain Res. 204, 217-225, doi:10.1016/j.bbr.2009.06.012 (2009).

12. Van Kampen, M. et al. AR-R 17779 improves social recognition in rats by activation of nicotinic alpha7 receptors. Psychopharmacology (Berl.) 172, 375-383, doi:10.1007/s00213-003-1668-7 (2004).

13. Wang, D. et al. Synergistic effect of galantamine with risperidone on impairment of social interaction in phencyclidine-treated mice as a schizophrenic animal model. Neuropharmacology 52, 1179-1187, doi:10.1016/j.neuropharm.2006.12.007 (2007).

14. Auld, D. S., Kornecook, T. J., Bastianetto, S. \& Quirion, R. Alzheimer's disease and the basal forebrain cholinergic system: relations to beta-amyloid peptides, cognition, and treatment strategies. Prog. Neurobiol. 68, 209-245 (2002).

15. Cerejeira, J., Lagarto, L. \& Mukaetova-Ladinska, E. B. Behavioral and psychological symptoms of dementia. Front. Neurol. 3, 73, doi:10.3389/fneur.2012.00073 (2012). 
16. Craig, L. A., Hong, N. S. \& McDonald, R. J. Revisiting the cholinergic hypothesis in the development of Alzheimer's disease. Neurosci. Biobehav. Rev. 35, 13971409, doi:10.1016/j.neubiorev.2011.03.001 (2011).

17. Ibach, B. \& Haen, E. Acetylcholinesterase inhibition in Alzheimer's Disease. Curr. Pharm. Des. 10, 231-251, doi:10.2174/1381612043386509 (2004).

18. Pinto, T., Lanctot, K. L. \& Herrmann, N. Revisiting the cholinergic hypothesis of behavioral and psychological symptoms in dementia of the Alzheimer's type. Ageing Res Rev 10, 404-412, doi:10.1016/j.arr.2011.01.003 (2011).

19. Ballinger, E. C., Ananth, M., Talmage, D. A. \& Role, L. W. Basal Forebrain Cholinergic Circuits and Signaling in Cognition and Cognitive Decline. Neuron 91, 1199-1218, doi:10.1016/j.neuron.2016.09.006 (2016).

20. Okada, K., Nishizawa, K., Kobayashi, T., Sakata, S. \& Kobayashi, K. Distinct roles of basal forebrain cholinergic neurons in spatial and object recognition memory. Sci. Rep. 5, 13158, doi:10.1038/srep13158 (2015).

21. Cummings, J. L. \& Kaufer, D. Neuropsychiatric aspects of Alzheimer's disease: the cholinergic hypothesis revisited. Neurology 47, 876-883, doi:10.1212/wnl.47.4.876 (1996).

22. Levy, M. L., Cummings, J. L. \& Kahn-Rose, R. Neuropsychiatric symptoms and cholinergic therapy for Alzheimer's disease. Gerontology 45 Suppl 1, 15-22, doi:10.1159/000052760 (1999).

23. Mesulam, M. M., Mufson, E. J., Wainer, B. H. \& Levey, A. I. Central cholinergic pathways in the rat: an overview based on an alternative nomenclature (Ch1-Ch6). Neuroscience 10, 1185-1201, doi:10.1016/0306-4522(83)90108-2 (1983). 
24. Woolf, N. J. Cholinergic systems in mammalian brain and spinal cord. Prog. Neurobiol. 37, 475-524, doi:10.1016/0301-0082(91)90006-M (1991).

25. Kobayashi, K. et al. Immunotoxin-mediated conditional disruption of specific neurons in transgenic mice. Proc. Natl. Acad. Sci. U. S. A. 92, 1132-1136, doi:10.1073/pnas.92.4.1132 (1995)

26. Kobayashi, K., Okada, K. \& Kai, N. in Controlled Genetic Manipulations (ed Alexei Morozov) 193-205 (Humana Press, 2012).

27. Kreitman, R. J. Recombinant immunotoxins containing truncated bacterial toxins for the treatment of hematologic malignancies. Biodrugs 23, 1-13, doi:10.2165/00063030-200923010-00001 (2009).

28. Wilkinson, D. G., Francis, P. T., Schwam, E. \& Payne-Parrish, J. Cholinesterase inhibitors used in the treatment of Alzheimer's disease: the relationship between pharmacological effects and clinical efficacy. Drugs Aging 21, 453-478, doi:10.2165/00002512-200421070-00004 (2004).

29. Boada-Rovira, M. et al. Efficacy and safety of donepezil in patients with Alzheimer's disease: results of a global, multinational, clinical experience study. Drugs Aging 21, 43-53, doi:10.2165/00002512-200421010-00004 (2004).

30. Rockwood, K., Graham, J. E. \& Fay, S. Goal setting and attainment in Alzheimer's disease patients treated with donepezil. J. Neurol. Neurosurg. Psychiatry 73, 500507, doi:10.1136/jnnp.73.5.500 (2002).

31. Yoo, J. H., Valdovinos, M. G. \& Williams, D. C. Relevance of donepezil in enhancing learning and memory in special populations: a review of the literature. $J$. Autism Dev. Disord. 37, 1883-1901, doi:10.1007/s10803-006-0322-8 (2007). 
32. Yuede, C. M., Dong, H. \& Csernansky, J. G. Anti-dementia drugs and hippocampal-dependent memory in rodents. Behav. Pharmacol. 18, 347-363, doi:10.1097/FBP.0b013e3282da278d (2007).

33. Johnson, D. A., Zambon, N. J. \& Gibbs, R. B. Selective lesion of cholinergic neurons in the medial septum by 192 IgG-saporin impairs learning in a delayed matching to position T-maze paradigm. Brain Res. 943, 132-141, doi:10.1016/s0006-8993(02)02623-9 (2002).

34. Lehmann, O., Grottick, A. J., Cassel, J. C. \& Higgins, G. A. A double dissociation between serial reaction time and radial maze performance in rats subjected to 192 IgG-saporin lesions of the nucleus basalis and/or the septal region. Eur. J.

Neurosci. 18, 651-666, doi:10.1046/j.1460-9568.2003.02745.x (2003).

35. Perry, T., Hodges, H. \& Gray, J. A. Behavioural, histological and immunocytochemical consequences following 192 IgG-saporin immunolesions of the basal forebrain cholinergic system. Brain Res. Bull. 54, 29-48, doi:10.1016/s0361-9230(00)00413-5 (2001).

36. Torres, E. M. et al. Behavioural, histochemical and biochemical consequences of selective immunolesions in discrete regions of the basal forebrain cholinergic system. Neuroscience 63, 95-122, doi:10.1016/0306-4522(94)90010-8 (1994).

37. Berger-Sweeney, J. et al. Selective immunolesions of cholinergic neurons in mice: effects on neuroanatomy, neurochemistry, and behavior. J. Neurosci. 21, 8164$8173(2001)$.

38. Hamlin, A. S., Windels, F., Boskovic, Z., Sah, P. \& Coulson, E. J. Lesions of the basal forebrain cholinergic system in mice disrupt idiothetic navigation. PLoS One 8, e53472, doi:10.1371/journal.pone.0053472 (2013). 
39. Moreau, P. H., Cosquer, B., Jeltsch, H., Cassel, J. C. \& Mathis, C. Neuroanatomical and behavioral effects of a novel version of the cholinergic immunotoxin mu p75saporin in mice. Hippocampus 18, 610-622, doi:10.1002/hipo.20422 (2008).

40. Lehmann, O. et al. 5,7-DHT-induced hippocampal 5-HT depletion attenuates behavioural deficits produced by 192 IgG-saporin lesions of septal cholinergic neurons in the rat. Eur. J. Neurosci. 15, 1991-2006, doi:10.1046/j.14609568.2002.02037.x (2002).

41. Lehmann, O. et al. Combined 192 IgG-saporin and 5,7-dihydroxytryptamine lesions in the male rat brain: a neurochemical and behavioral study. Pharmacol. Biochem. Behav. 72, 899-912, doi:10.1016/s0091-3057(02)00752-9 (2002).

42. van der Staay, F. J. et al. Long-term effects of immunotoxic cholinergic lesions in the septum on acquisition of the cone-field task and noncognitive measures in rats. Hippocampus 16, 1061-1079, doi:10.1002/hipo.20229 (2006).

43. Kitabatake, Y., Hikida, T., Watanabe, D., Pastan, I. \& Nakanishi, S. Impairment of reward-related learning by cholinergic cell ablation in the striatum. Proc. Natl. Acad. Sci. U. S. A. 100, 7965-7970, doi:10.1073/pnas.1032899100 (2003).

44. Sano, H. et al. Conditional ablation of striatal neuronal types containing dopamine D2 receptor disturbs coordination of basal ganglia function. J. Neurosci. 23, 90789088, doi:10.1523/jneurosci.23-27-09078.2003 (2003).

45. Ogura, H., Kosasa, T., Kuriya, Y. \& Yamanishi, Y. Comparison of inhibitory activities of donepezil and other cholinesterase inhibitors on acetylcholinesterase and butyrylcholinesterase in vitro. Methods Find. Exp. Clin. Pharmacol. 22, 609$613(2000)$. 
46. Darvesh, S., Hopkins, D. A. \& Geula, C. Neurobiology of butyrylcholinesterase. Nat. Rev. Neurosci. 4, 131-138, doi:10.1038/nrn1035 (2003).

47. Tago, H., Maeda, T., McGeer, P. L. \& Kimura, H. Butyrylcholinesterase-rich neurons in rat brain demonstrated by a sensitive histochemical method. J. Comp. Neurol. 325, 301-312, doi:10.1002/cne.903250212 (1992).

48. Alexander, G. E., DeLong, M. R. \& Strick, P. L. Parallel organization of functionally segregated circuits linking basal ganglia and cortex. Annu. Rev. Neurosci. 9, 357-381, doi:10.1146/annurev.ne.09.030186.002041 (1986).

49. Nelson, A. B. \& Kreitzer, A. C. Reassessing models of basal ganglia function and dysfunction. Annu. Rev. Neurosci. 37, 117-135, doi:10.1146/annurev-neuro071013-013916 (2014).

50. Li, X. et al. Generation of a whole-brain atlas for the cholinergic system and mesoscopic projectome analysis of basal forebrain cholinergic neurons. Proc. Natl. Acad. Sci. U. S. A. 115, 415-420, doi:10.1073/pnas.1703601115 (2018).

51. Deacon, R. M., Bannerman, D. M. \& Rawlins, J. N. Anxiolytic effects of cytotoxic hippocampal lesions in rats. Behav. Neurosci. 116, 494-497, doi:10.1037//07357044.116.3.494 (2002).

52. McHugh, S. B., Deacon, R. M., Rawlins, J. N. \& Bannerman, D. M. Amygdala and ventral hippocampus contribute differentially to mechanisms of fear and anxiety. Behav. Neurosci. 118, 63-78, doi:10.1037/0735-7044.118.1.63 (2004).

53. Avale, M. E. et al. Prefrontal nicotinic receptors control novel social interaction between mice. FASEB J. 25, 2145-2155, doi:10.1096/fj.10-178558 (2011).

54. Savage, S., Kehr, J., Olson, L. \& Mattsson, A. Impaired social interaction and enhanced sensitivity to phencyclidine-induced deficits in novel object recognition 
in rats with cortical cholinergic denervation. Neuroscience 195, 60-69, doi:10.1016/j.neuroscience.2011.08.027 (2011).

55. Prado, V. F. et al. Mice deficient for the vesicular acetylcholine transporter are myasthenic and have deficits in object and social recognition. Neuron 51, 601-612, doi:10.1016/j.neuron.2006.08.005 (2006).

56. Winslow, J. T. \& Camacho, F. Cholinergic modulation of a decrement in social investigation following repeated contacts between mice. Psychopharmacology (Berl.) 121, 164-172, doi:10.1007/bf02245626 (1995).

57. Wenk, G. L. The Nucleus Basalis Magnocellularis Cholinergic System: One Hundred Years of Progress. Neurobiol. Learn. Mem. 67, 85-95, doi:https://doi.org/10.1006/nlme.1996.3757 (1997).

58. Tanimizu, T. et al. Functional Connectivity of Multiple Brain Regions Required for the Consolidation of Social Recognition Memory. J. Neurosci. 37, 4103-4116, doi:10.1523/jneurosci.3451-16.2017 (2017).

59. Katona, C. et al. International Psychogeriatric Association consensus statement on defining and measuring treatment benefits in dementia. Int. Psychogeriatr. 19, 345354, doi:10.1017/s1041610207005145 (2007).

60. Paxinos, G. \& Franklin, K. B. J. The mouse brain in stereotaxic coordinates. (Academic Press, 2001).

\section{Acknowledgements}

This work was supported by Grants-in-Aid for Scientific Research C (\#18K03178) from the Ministry of Education, Science, Sports and Culture of Japan (K.O.); and Core 
Research for Evolutional Science and Technology of Japan Science and Technology Agency (K.K.), and by Career Assistant Project in Hiroshima University (K.O.). It was also funded by Novartis Pharma K.K., Japan and Ono Pharmaceutical Co, Ltd (K.K.). We thank Dr. I. Pastan for providing anti-Tac(Fv)-PE38. We are also grateful to M. Kikuchi, N. Sato and K. Sato for their technical support in animal experiments, as well as N. Demura (Novartis Pharma K.K.) for his scientific discussion.

\section{Author contributions}

K.O., S.S., K.H. and K.K. conceived the study, designed the experiments, and directed the project. K.O., K.N., and T. K. performed intracranial injection and histological examination. K.O. and K.K designed and performed the behavioural analyses. K.O. and K.K. wrote the paper. All authors discussed the results and implications, and commented on the manuscript at all stages.

\section{Additional Information}

\section{Competing financial interests}

The authors declare no competing financial interests.

\section{Data availability}

The datasets generated and analyzed in the current study will be registered in the Mendeley data repository after the acceptance of the manuscript. 


\section{Figures and figure legends}

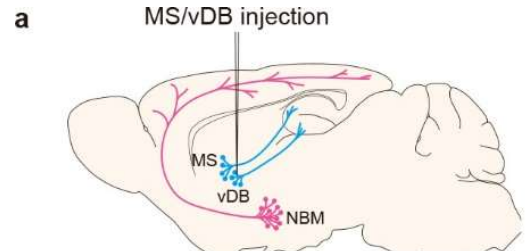

C
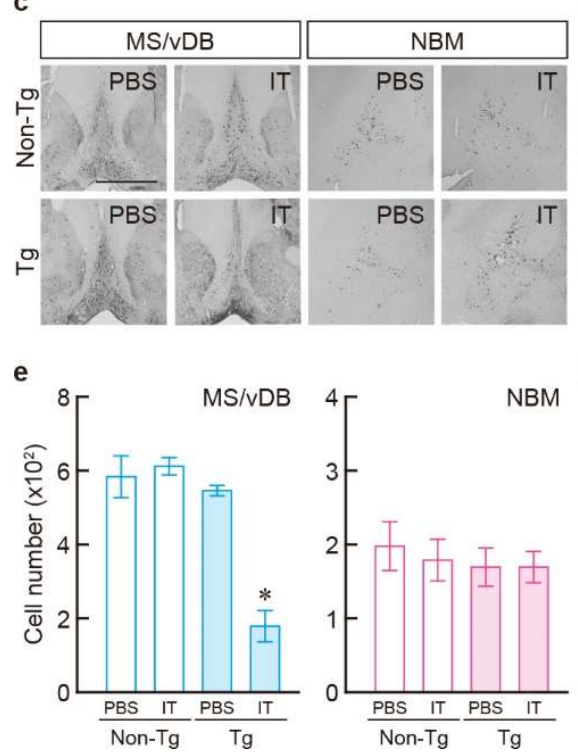

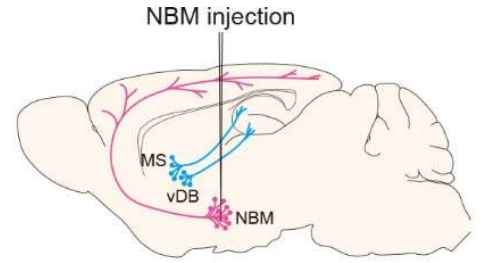

d
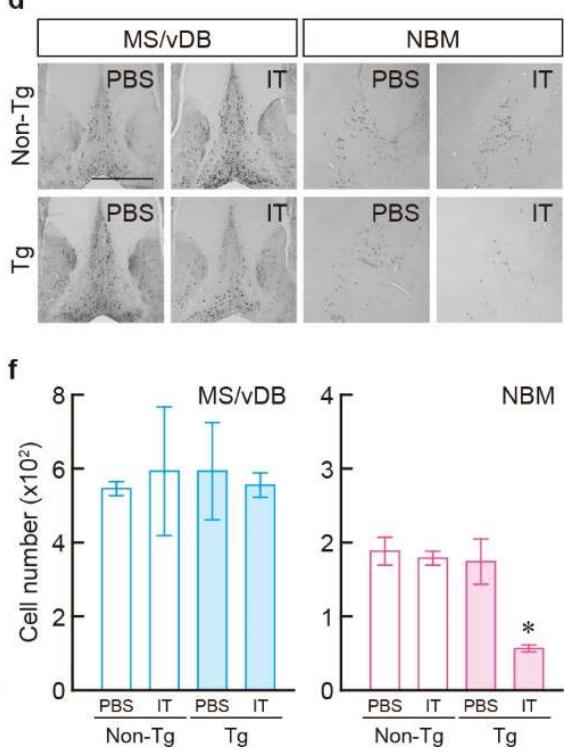

NBM

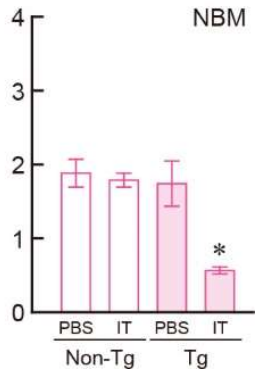

Figure 1. Selective targeting of basal forebrain cholinergic cell groups. (a,b)

Schematic representation of the intracranial IT injection by using stereotaxic surgery into the MS/vDB (a) or NBM (b). Black needles indicate the glass pipets for the injection. (c,d) ChAT immunostaining with sections through the MS/vDB or NBM prepared from the Tg and non-Tg mice 7 days after IT/PBS injection into the MS/vDB (c) or NBM (d). Scale bars: $1 \mathrm{~mm}$. (e, f) Cell counts of ChAT-positive neurons in the MS/vDB or NBM in the injected mice into the MS/vDB (e) or NBM (f). Data are presented as mean \pm s.e.m. $n=3$ for each group. ${ }^{*} P<0.05$ vs each of other three groups (Bonferroni method). 

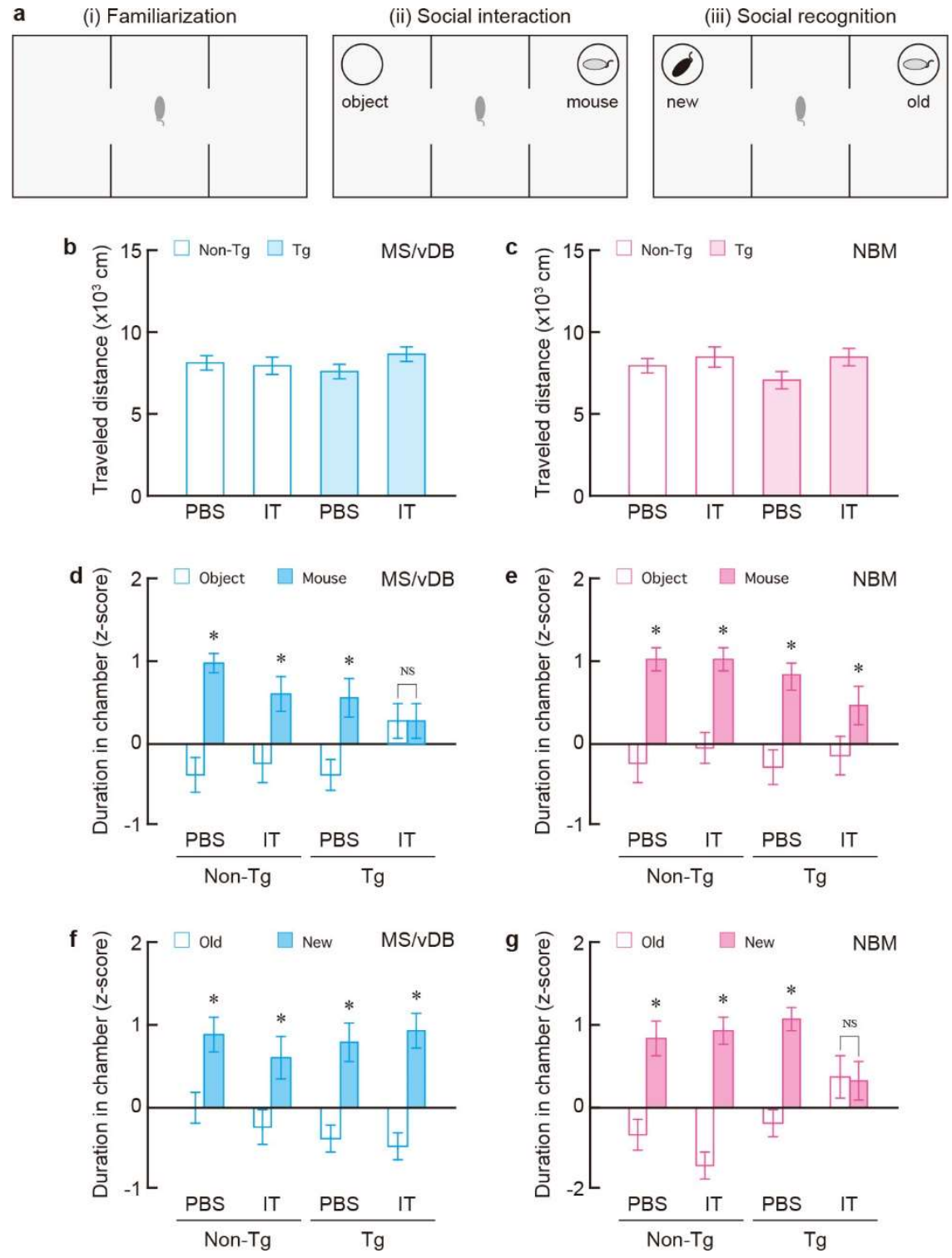

\section{Figure 2. Elimination of the MS/vDB and NBM cholinergic neurons impaired} social interaction and social recognition memory, respectively. $\mathrm{Tg}$ and non- $\mathrm{Tg}$ mice were injected with IT solution or PBS into the MS/vDB or NBM and then performed for the behavioural test. (a) Strategy for the three-chamber test. The large square represents the rectangle open field, which was divided into three chambers (a central chamber and two side chambers). The bold circled areas indicate the cages. The mice individually partook in the three successive 10-min sessions for (i) familiarization, (ii) 
social interaction, and (iii) social recognition memory, with 1-min intersession intervals within one day. (b,c) Mean travelled distance in the apparatus during the familiarization session in the injected mice into the MS/vDB (b) or NBM (c). Data are presented as mean \pm s.e.m. $n=15$ for each group. (d,e) Mean z-score of the duration in the chambers with the mouse and the object cages during the social interaction session in the injected mice into the MS/vDB (d) or NBM (e). Data are presented as mean \pm s.e.m. $n=15$ for each group. ${ }^{*} P<0.05$ vs the object chamber (two-way ANOVA with the repeated measure). NS, not significant. (f,g) Mean z-score of the duration in the chambers with familiar mouse (old) and novel mouse (new) during the social recognition memory session in the injected mice into the MS/vDB (f) or NBM (g). Data are presented as mean \pm s.e.m. $n=15$ for each group. $* P<0.05$ vs the old mouse chamber (two-way ANOVA with the repeated measure). NS, not significant. 
a

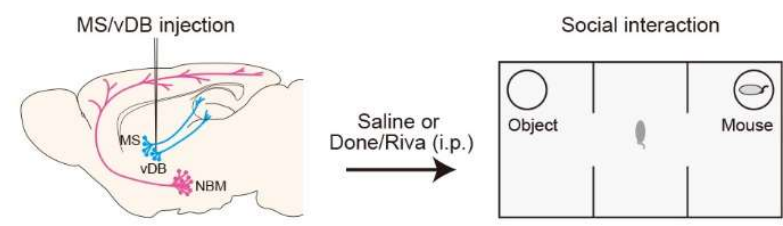

b

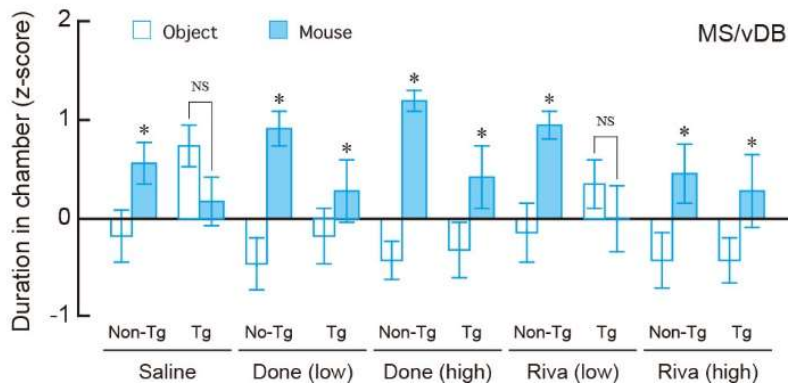

Figure 3. Drug effect on the deficit in social interaction in mice lacking MS/vDB cholinergic neurons. (a) Experimental design for a recovery experiment from the interaction deficit. Tg and non-Tg mice injected with IT solution into the MS/vDB were given systemic administration (i.p.) with saline, Done or Riva at low and high doses (1 and $4 \mu \mathrm{mol} / \mathrm{kg}$, respectively), and $30 \mathrm{~min}$ later were used for the three-chamber test. (b) Mean z-score of the duration in the object and mouse chambers during the social interaction session. Data are presented as mean \pm s.e.m. $n=10$ for each group. ${ }^{*} P<$ 0.05 vs the object chamber (two-way ANOVA with the repeated measure). NS, not significant. 
a

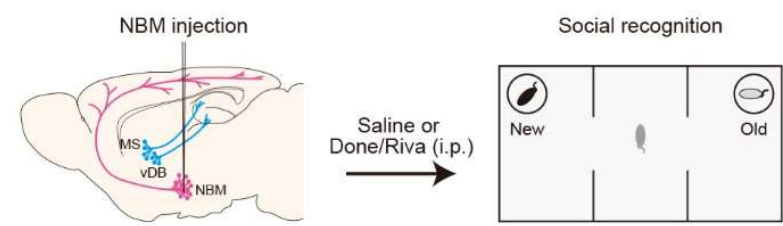

b

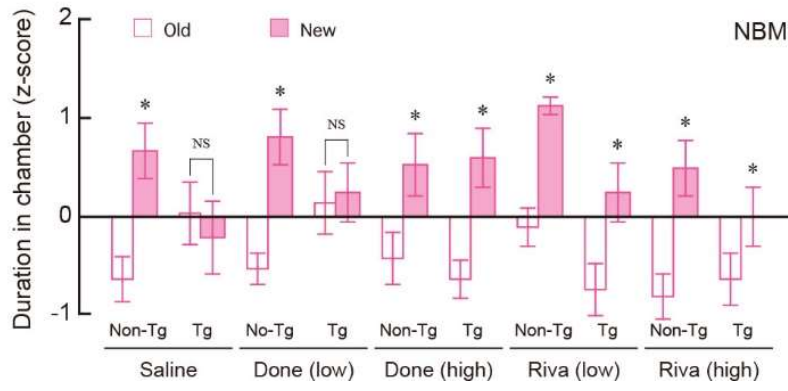

Figure 4. Drug influence on the impairment in social recognition memory in mice lacking NBM cholinergic neurons. (a) Experimental design for a recovery experiment from the memory impairment. Tg and non-Tg mice with IT injection into the NBM were administered i.p. with saline, Done or Riva with the low and high doses ( 1 and 4 $\mu \mathrm{mol} / \mathrm{kg}$, respectively), and then 30 min later subjected to the three-chamber test. (b) Mean z-score of the duration in the old and new mouse chambers during the social recognition memory session. Data are presented as mean \pm s.e.m. $n=10$ for each group. ${ }^{*} P<0.05$ vs the old mouse chamber (two-way ANOVA with the repeated measure). NS, not significant. 

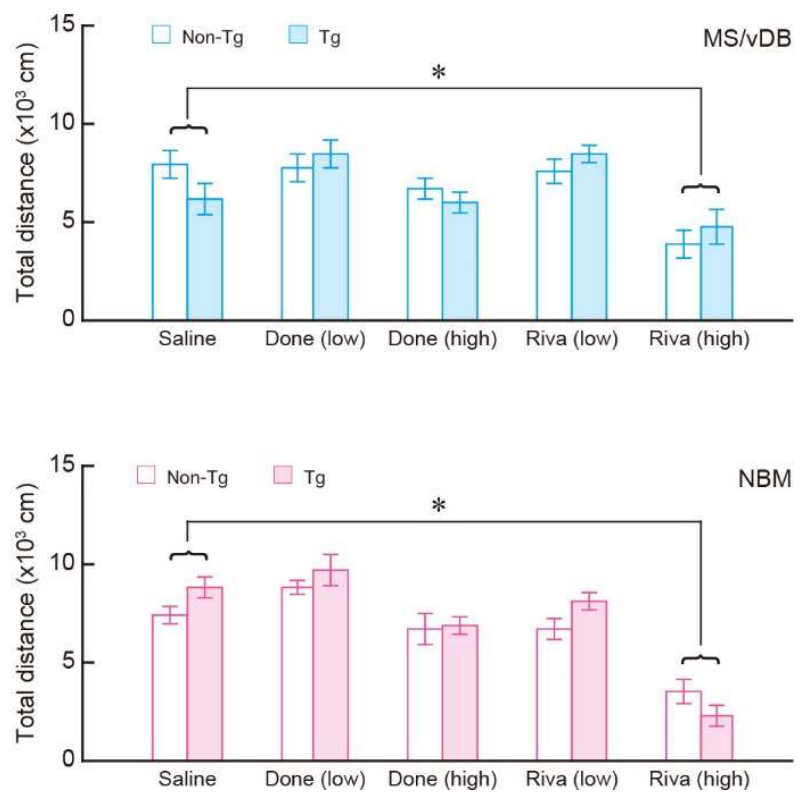

Figure 5. Locomotor activity in mice lacking basal forebrain cholinergic neurons after drug administration. Mice with IT injection into the MS/vDB or NBM were administered i.p. with saline, Done or Riva at low and high doses ( 1 and $4 \mu \mathrm{mol} / \mathrm{kg}$, respectively), and 30 min later were moved to the familiarization session in the threechamber test. $(\mathbf{a}, \mathbf{b})$ Mean travelled distance in the apparatus during the familiarization session in the injected mice into the MS/vDB (a) or NBM (b). Data are presented as mean \pm s.e.m. $n=10$ for each group. ${ }^{*} P<0.05$ vs saline administration (Bonferroni method). 


\section{Figures}

a

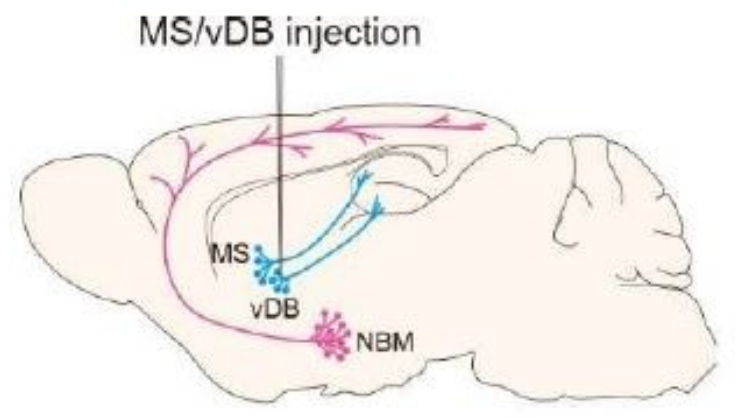

C
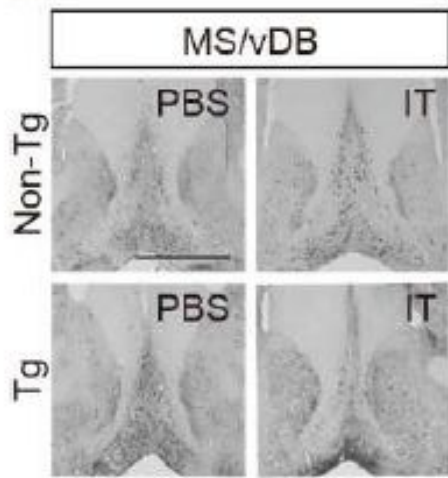

e

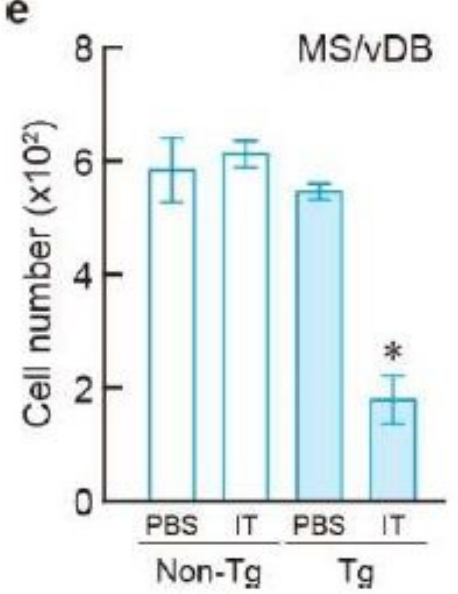

b

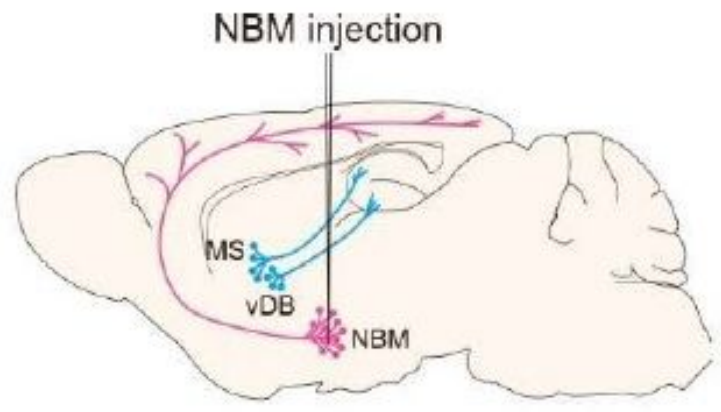

d
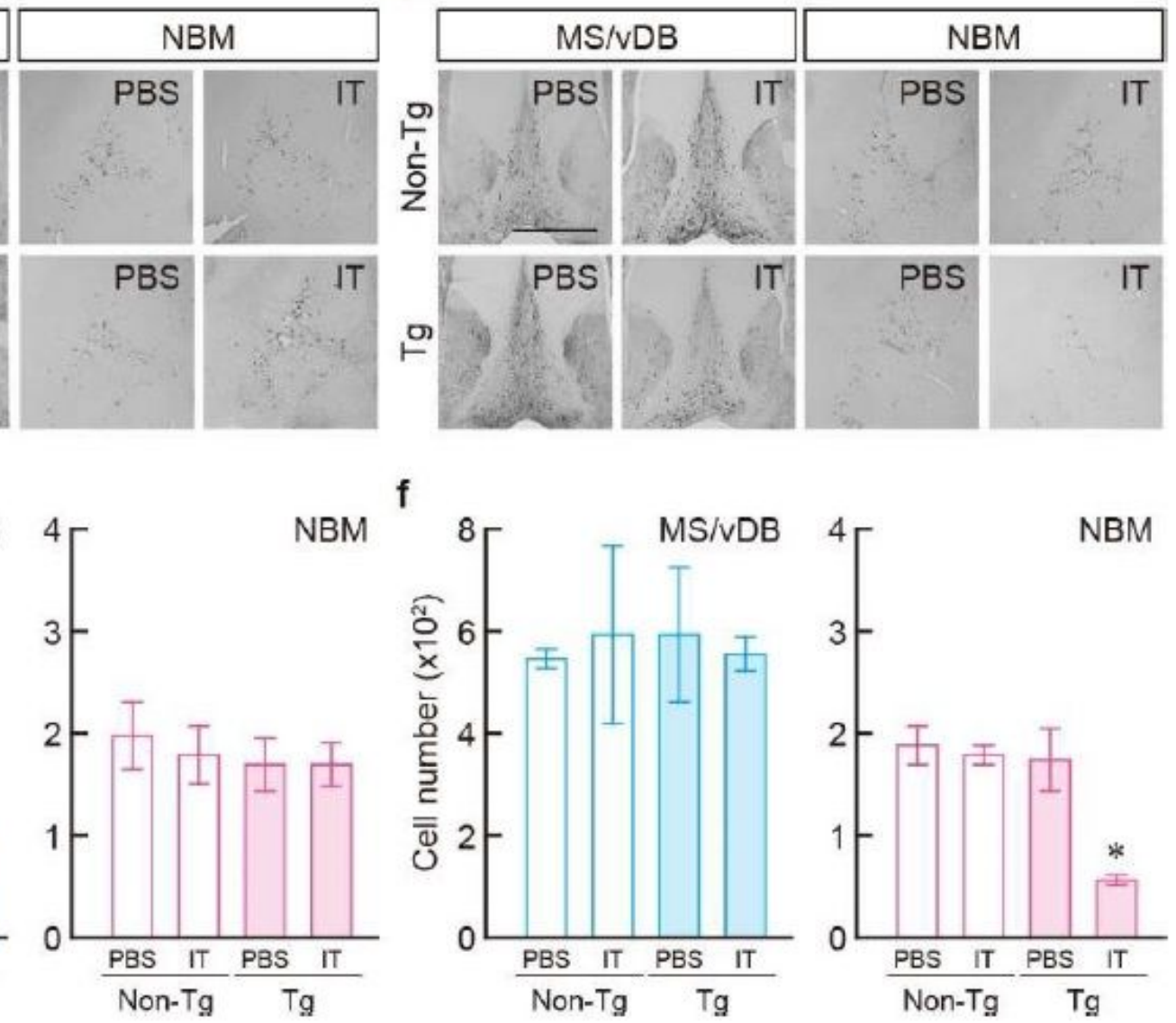

\section{Figure 1}

Selective targeting of basal forebrain cholinergic cell groups. $(a, b)$ Schematic representation of the intracranial IT injection by using stereotaxic surgery into the MS/vDB (a) or NBM (b). Black needles indicate the glass pipets for the injection. $(c, d)$ ChAT immunostaining with sections through the MS/vDB or NBM prepared from the Tg and non-Tg mice 7 days after IT/PBS injection into the MS/vDB c) or NBM (d). Scale bars: $1 \mathrm{~mm}$. (e, f) Cell counts of ChAT-positive neurons in the MS/vDB or NBM in the injected mice into the MS/vDB (e) or NBM (f). Data are presented as mean \pm s.e.m. $n=3$ for each group. ${ }^{\star} \mathrm{P}<0.05$ vs each of other three groups (Bonferroni method). 
a

(i) Familiarization

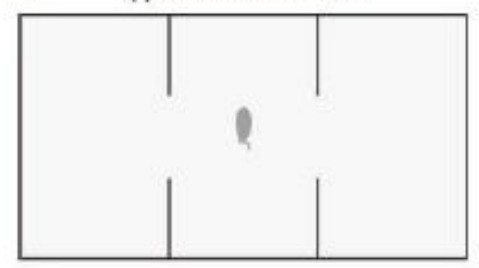

b
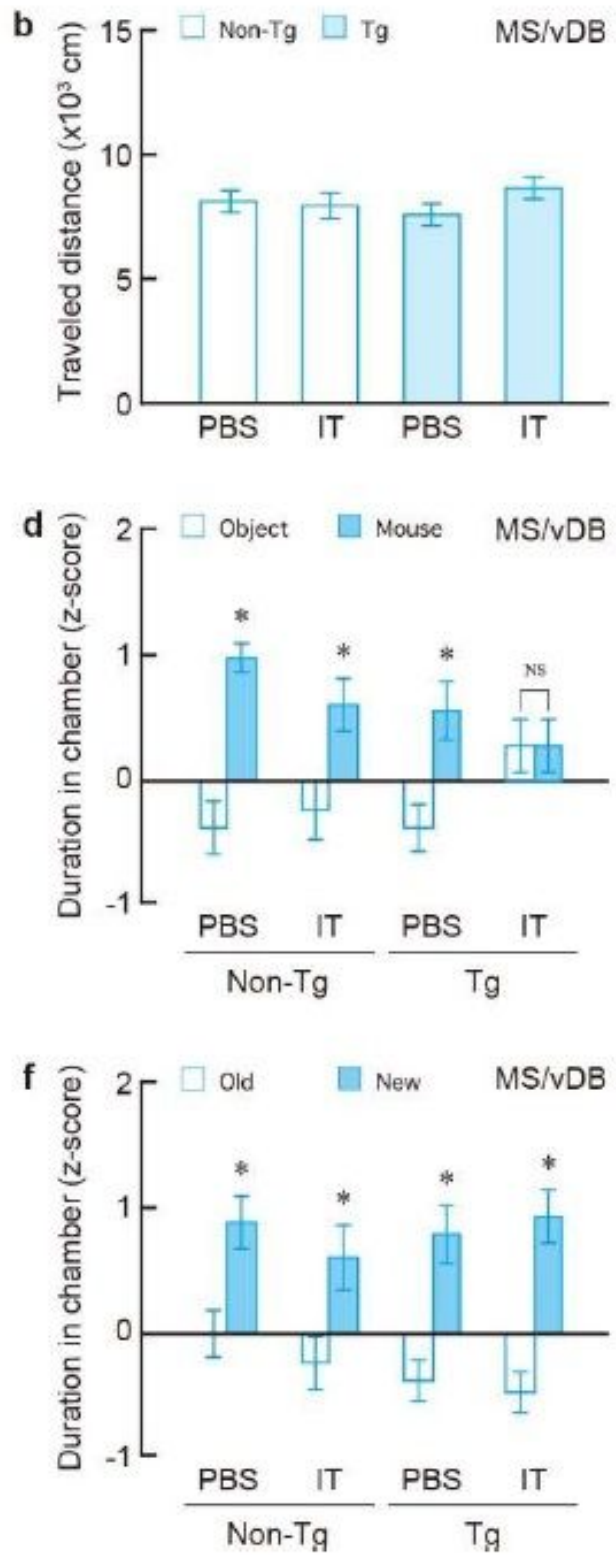

(ii) Social interaction

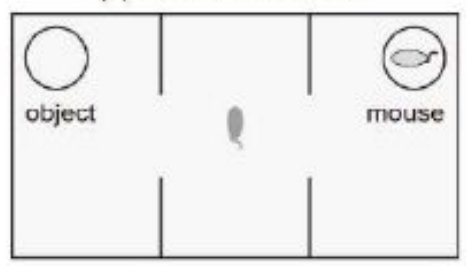

c ${ }^{15} \square$ Non-Tg $\square \mathrm{Tg}$

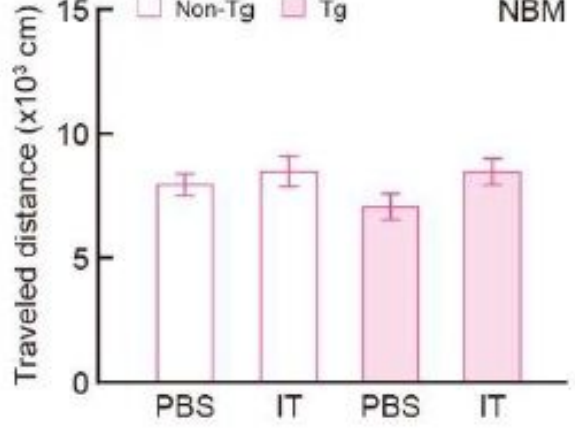

NBM
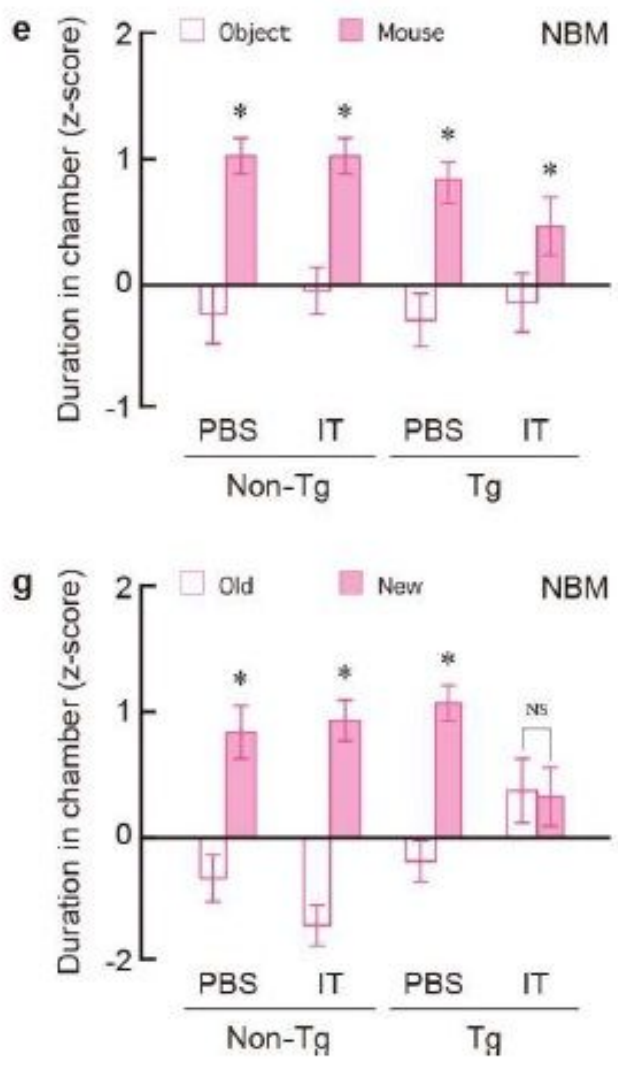

\section{Figure 2}

Elimination of the MS/vDB and NBM cholinergic neurons impaired social interaction and social recognition memory, respectively. Tg and non-Tg mice were injected with IT solution or PBS into the MS/vDB or NBM and then performed for the behavioural test. (a) Strategy for the three-chamber test. The large square represents the rectangle open field, which was divided into three chambers (a central chamber and two side chambers). The bold circled areas indicate the cages. The mice individually 
partook in the three successive 10-min sessions for (i) familiarization, (ii) social interaction, and (iii) social recognition memory, with 1-min intersession intervals within one day. $(b, c)$ Mean travelled distance in the apparatus during the familiarization session in the injected mice into the MS/vDB (b) or NBM (c). Data are presented as mean \pm s.e.m. $n=15$ for each group. $(d, e)$ Mean z-score of the duration in the chambers with the mouse and the object cages during the social interaction session in the injected mice into the MS/vDB (d) or NBM (e). Data are presented as mean \pm s.e.m. $n=15$ for each group. ${ }^{*} P<0.05$ vs the object chamber (two-way ANOVA with the repeated measure). NS, not significant. $(f, g)$ Mean z-score of the duration in the chambers with familiar mouse (old) and novel mouse (new) during the social recognition memory session in the injected mice into the MS/vDB (f) or NBM (g). Data are presented as mean \pm s.e.m. $n=15$ for each group. ${ }^{*} P<0.05$ vs the old mouse chamber (two-way ANOVA with the repeated measure). NS, not significant.

a
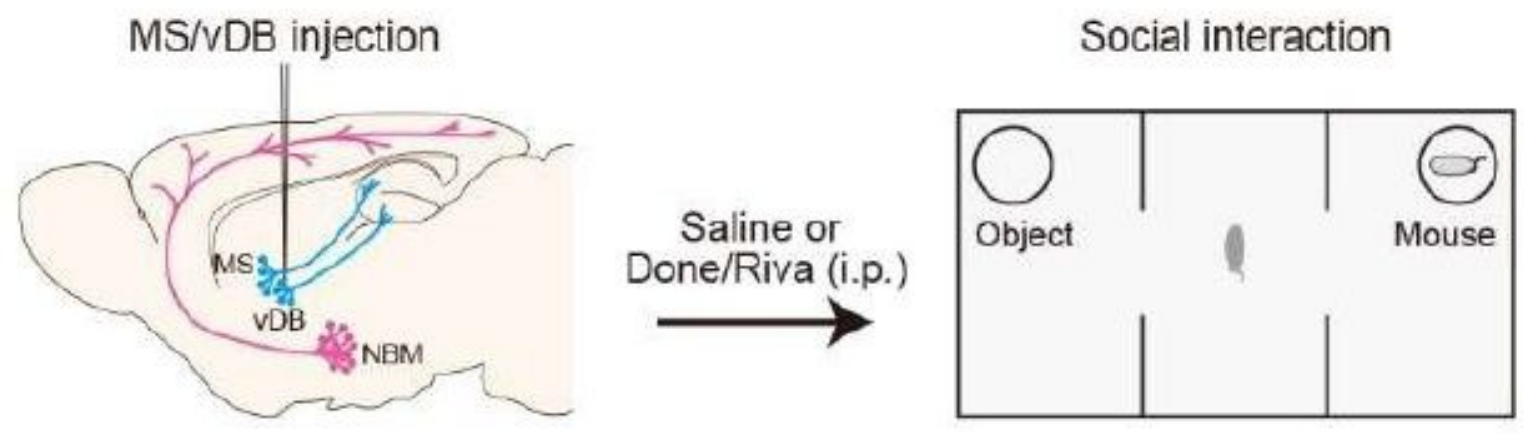

b

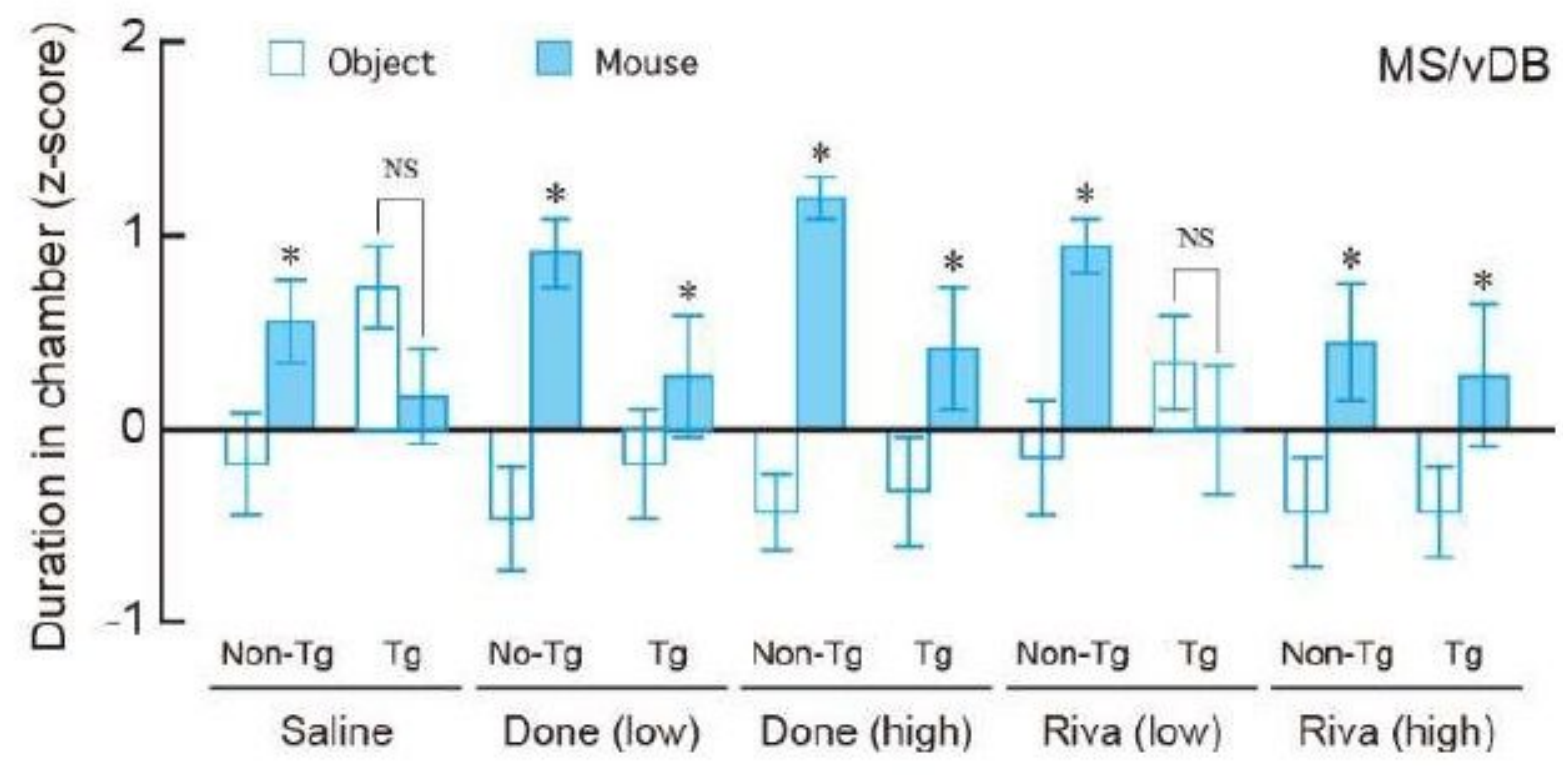

Figure 3 
Drug effect on the deficit in social interaction in mice lacking MS/vDB cholinergic neurons. (a) Experimental design for a recovery experiment from the interaction deficit. Tg and non-Tg mice injected with IT solution into the MS/vDB were given systemic administration (i.p.) with saline, Done or Riva at low and high doses ( 1 and $4 \mu \mathrm{mol} / \mathrm{kg}$, respectively), and $30 \mathrm{~min}$ later were used for the three-chamber test. (b) Mean z-score of the duration in the object and mouse chambers during the social interaction session. Data are presented as mean \pm s.e.m. $n=10$ for each group. ${ }^{*} P<0.05$ vs the object chamber (twoway ANOVA with the repeated measure). NS, not significant.

a
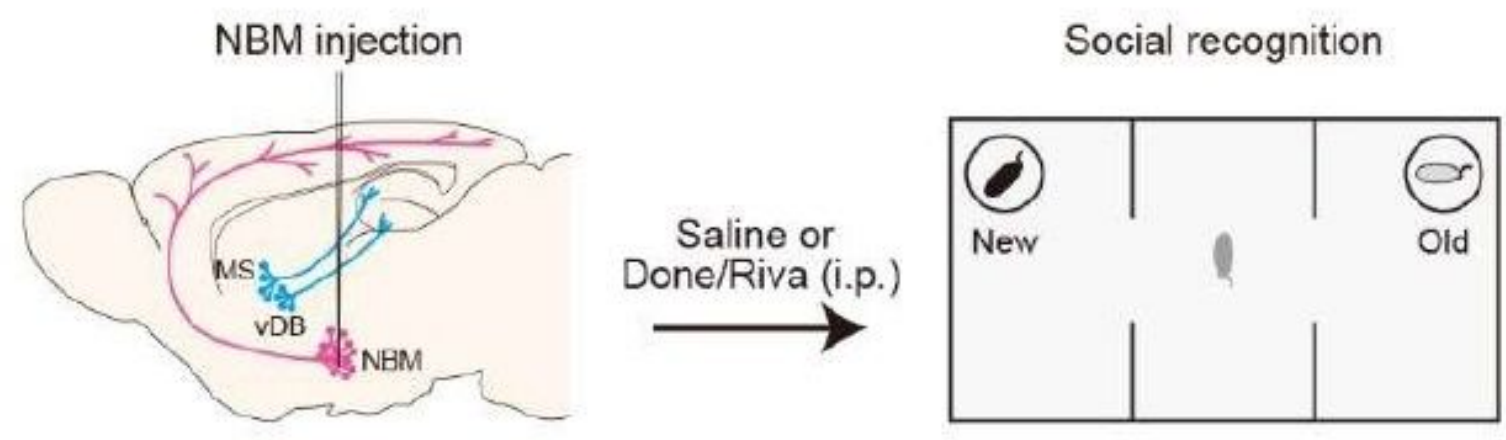

b

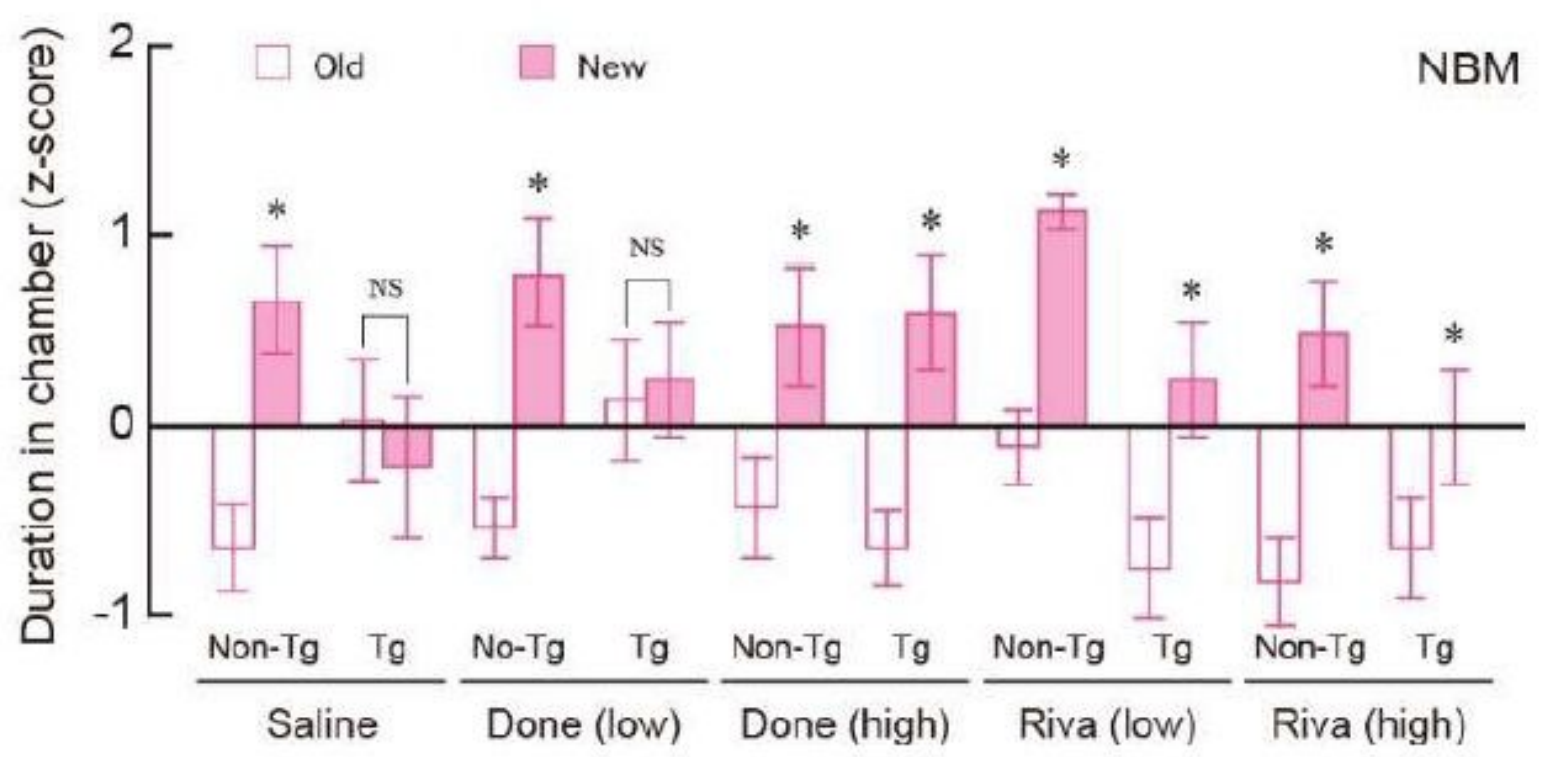

Figure 4

Drug influence on the impairment in social recognition memory in mice lacking NBM cholinergic neurons. (a) Experimental design for a recovery experiment from the memory impairment. Tg and non-Tg mice with IT injection into the NBM were administered i.p. with saline, Done or Riva with the low and high doses (1 and $4 \mu \mathrm{mol} / \mathrm{kg}$, respectively), and then $30 \mathrm{~min}$ later subjected to the three-chamber test. (b) Mean z-score of the duration in the old and new mouse chambers during the social recognition memory session. Data 
are presented as mean \pm s.e.m. $n=10$ for each group. ${ }^{*} P<0.05$ vs the old mouse chamber (two-way ANOVA with the repeated measure). NS, not significant.

a

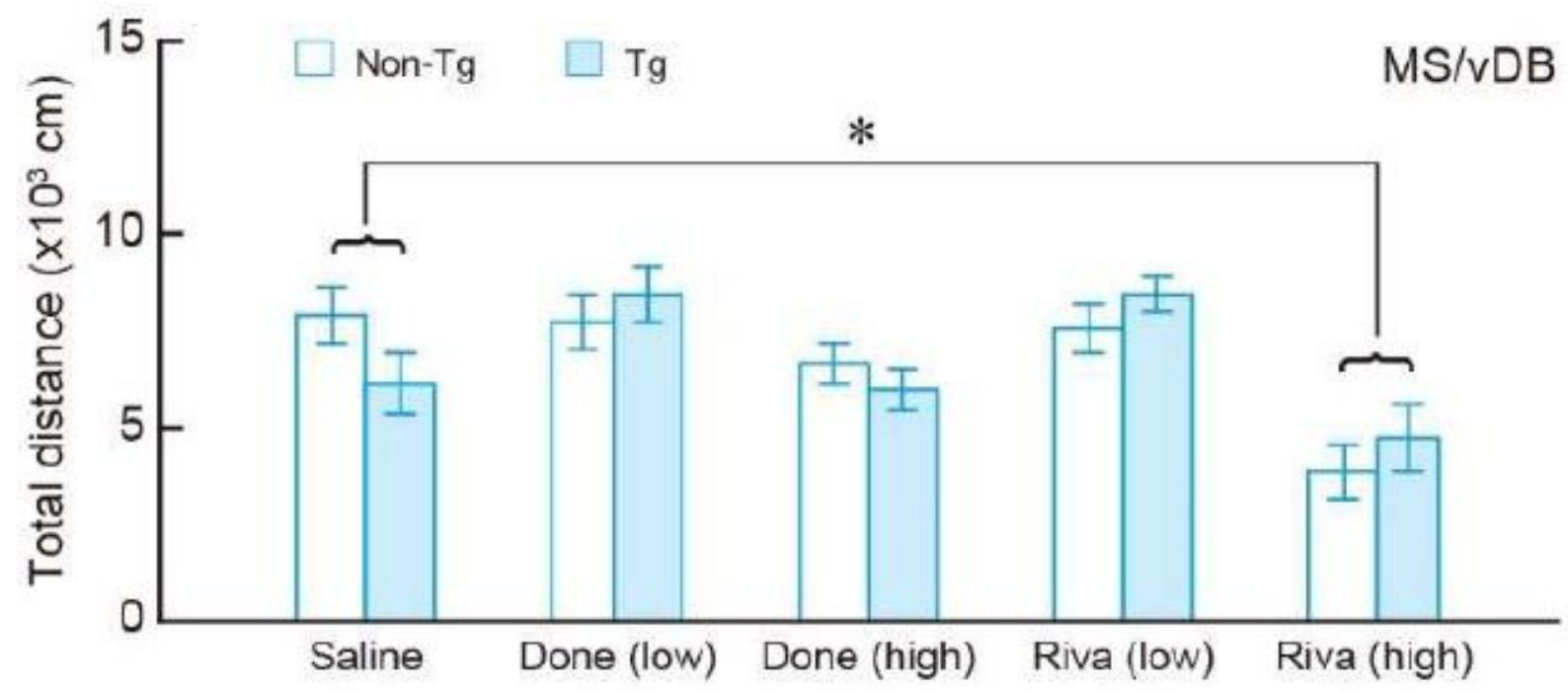

b

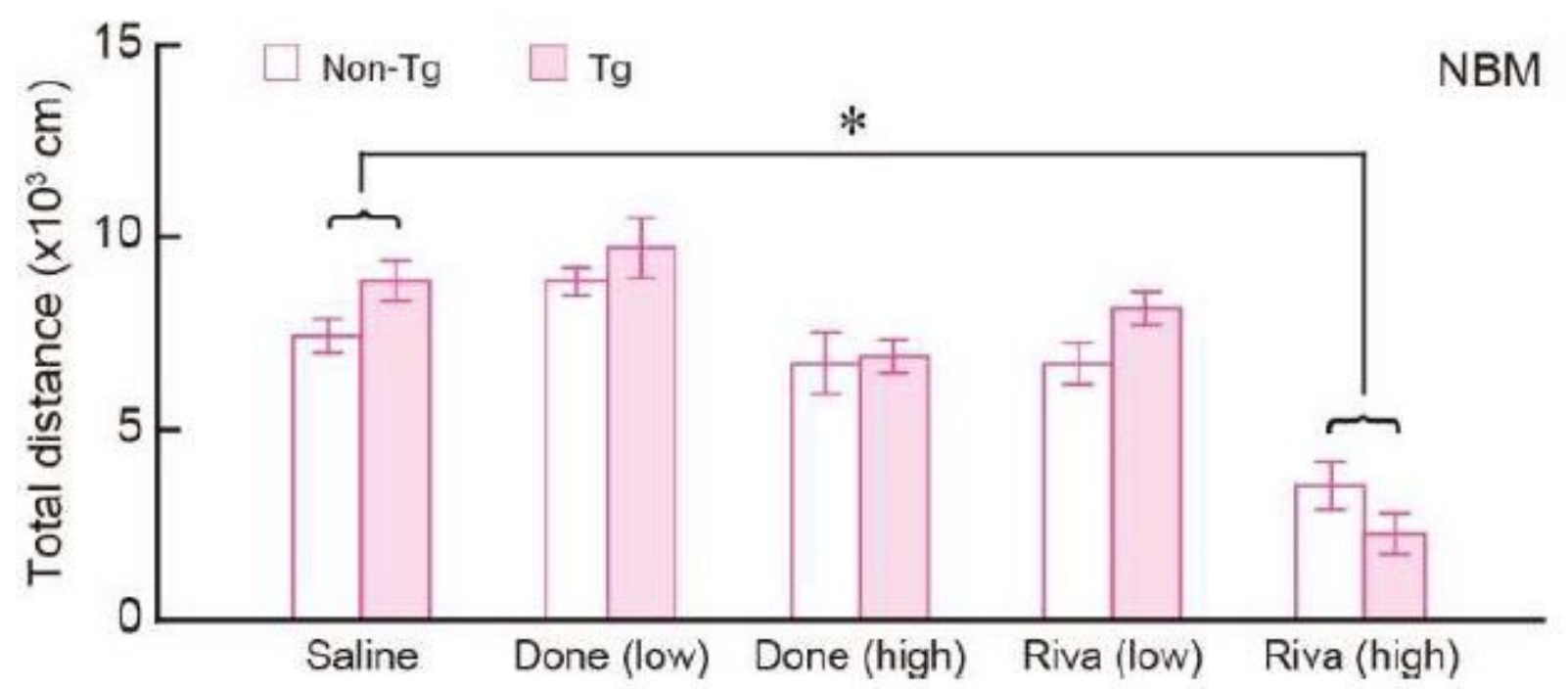

Figure 5

Locomotor activity in mice lacking basal forebrain cholinergic neurons after drug administration. Mice with IT injection into the MS/vDB or NBM were administered i.p. with saline, Done or Riva at low and high doses ( 1 and $4 \mu \mathrm{mol} / \mathrm{kg}$, respectively), and $30 \mathrm{~min}$ later were moved to the familiarization session in the threechamber test. $(a, b)$ Mean travelled distance in the apparatus during the familiarization session in the 
injected mice into the MS/vDB (a) or NBM (b). Data are presented as mean \pm s.e.m. $n=10$ for each group. ${ }^{*} P<0.05$ vs saline administration (Bonferroni method). 